\title{
Vorwort
}

Mit der vorliegenden Publikation gibt die Landesstelle für die nichtstaatlichen Museen den Museumskolleginnen und -kollegen eine Arbeitshilfe an die Hand, die das umfangreiche Thema »Museum und Digitales« beleuchtet und für die praktische Arbeit handhabbar macht. Der Band dokumentiert aktuelle Technologien bzw. Strategien und stellt wichtige Instrumente in den Arbeitsbereichen Dokumentation, Kommunikation und Vermittlung vor. Zudem gibt er Orientierungen und Anregungen zum Betrieb digitaler Medien im Museum und bietet Hilfestellungen zu zahlreichen Themen der Digitalisierung wie Webseiten, Online-Sammlungen, Social Media, Medienstationen, Medienguides, E-Publishing, Storytelling oder digitale Strategien.

\section{Konzeptteam und Autoren}

Viele haben zum Gelingen des Buchprojekts und seiner digitalen Erweiterungen beigetragen. Zunächst muss Dr. Regina Franken-Wendelstorf genannt werden, die als Ideengeberin für die thematische Ausrichtung des Buches fungierte. Dank ihrer Erfahrung in der wissenschaftlichen Begleitung praxisorientierter Forschungsprojekte zum Medieneinsatz in Museen wurde es möglich, das Thema "Das erweiterte Museum « möglichst umfassend darzustellen. Wichtige Facetten und eine Menge Expertise bringen zwei Hauptautoren mit ein: Sybille Greisinger, bei der Landesstelle unter anderem verantwortlich für den Bereich Digitale Kommunikation, und Dr. Christian Gries, Leiter des Landesstellenprojekts »Digitale Strategien für Museen«. Darüber hinaus hat das Herausgeber- und Autorenteam weitere Fachleute für ausgewählte Themen gewinnen können. Ihre Beiträge sind jeweils mit den Autorennamen gekennzeichnet. Barbara Kappelmayr hat im Lektorat dafür Sorge getragen, dass die Texte der verschiedenen Autorinnen und Autoren zu einem Buch »wie aus einem Guss» wurden.

\section{Ein aktuelles Thema - drei Publikationsformate}

Es scheint geradezu anachronistisch, über Apekte der Digitalisierung eine gedruckte Publikation zu produzieren. Dieser Aufgabenbereich ist durch eine extreme Dynamik gekennzeichnet und die eine oder andere Betrachtung ist am Erscheinungstag vielleicht schon veraltet. Um diesem Dilemma zu begegnen, haben wir uns von Beginn an für ein dreigliedriges Publikationskonzept entschieden: das gedruckte Buch, das E-Book und die korrespondierende Webseite - das »dynamische Projekt-Portal«, das es uns erlaubt, Neuerungen zu publizieren (vgl. nebenstehende Grafik). Während Buch und E-Book dieselben Inhalte auf unterschiedlichen Medien transportieren, versteht sich die digitale Erweiterung als »living document", als ein lebendiges Format, das über QR-Codes mit der Printversion sowie über Verlinkungen mit dem E-Book verknüpft ist. Dieses in Zusammenarbeit mit der Bayerischen 
Staatsbibliothek entwickelte, nachhaltig redaktionell betreute Portal erlaubt es uns, das Thema laufend zu erweitern und damit auch künftig für die Museumsarbeit aktuell vorzuhalten. Die Entscheidung für die dreigegliederte Publikation ist aber nicht nur der Schnelllebigkeit des Themas geschuldet. Vielmehr möchten wir als Landesstelle auch in unserer Vorbildfunktion mit neuen Publikationsformaten vorangehen. Wenn wir die Museen ermuntern, sich dem Digitalen konsequent und systematisch zu öffnen, ist es selbstverständlich, dass wir uns auch selbst auf den Weg begeben. So hat uns das Projekt nicht nur unser erstes E-Book und eine klare Position im wichtigen Themenbereich Open Access beschert, sondern gerade auch mit dem dynamischen Projekt-Portal ein zukunftsweisendes Kooperationsprojekt mit der Bayerischen Staatsbibliothek eröffnet. Auch dieses Prinzip verstehen wir gerne als Empfehlung an die Museen: Man muss und kann nicht alles alleine auf die Beine stellen. Der richtige Partner an der Seite eröffnet neue Wege und Horizonte.

Und auch das Erscheinungsbild dieses Bandes ist neu. Wir alle nutzen zunehmend Informations- und Wissensangebote im Internet. Digitale Angebote werden nur dann rezipiert, wenn sie klar strukturiert und knapp gehalten sind. Dies bleibt wiederum nicht ohne Auswirkungen auf das Leseverhalten bei Printprodukten. So möchten wir mit einem aktualisierten Layout und einer noch besseren Strukturierung unsere MuseumsBausteine optimieren und damit auch die stets knappen Zeitressourcen von Museumsmitarbeitern berücksichtigen.

\section{Dynamisches Projekt-Portal}

In der Zusammenarbeit mit der Bayerischen Staatsbibliothek München ist das dynamische Projekt-Portal (dynamic publication system/dyps) als digitale Erweiterung des Buches entstanden, die vor allem den sich rasch wandelnden Themen mit Best-Practice-Beispielen, Linktipps und Arbeitshilfen Rechnung tragen will. Unter dem Begriff »dyps « entwickelte das Zentrum für Elektronisches Publizieren der Bayerischen Staatsbibliothek eine Anwendung, die dynamische multimediale Dokumente möglichst unabhängig vom Endgerät anzeigt und nutzbar macht. Dabei wird der Volltext des Dokuments angereichert mit Bildern, Multimedia-Objekten sowie Auszügen aus digitalen Datenbanken und archivierten Webseiten. Alle Bestandteile des Dokuments sind entsprechend dem inhaltlichen Zusammenhang verknüpft und kommentiert. Gleichzeitig eröffnet diese Konzeption auch neue Potenziale für veränderliche Dokumente, deren einzelne Absätze, Bilder oder sonstige Komponenten trotz einer Aktualisierung nachvollziehbar und zitierbar sind. Das Projekt strebt nach einer quelloffenen Lösung, die möglichst niedrige Anforderungen an die Endgeräte der Nutzer stellt. Die Publikation erscheint mit einer offenen Lizenz: Alle Texte stehen unter der Creative-Commons-Lizenz CC BY 4.0 International (Namensnennung) und können entsprechend weitergenutzt werden. Alle publizierten Angebote sind unter www.mb-19.de zur Verfügung gestellt. 


\section{Lesehinweise}

Die Digitalisierung führt eine Menge neuer Begriffe in den Sprachgebrauch ein. Wir haben uns bei der Erstellung der Texte um eine möglichst klare Terminologie und verständliche Sprache bemüht, die auch digitale Einsteiger auf das Thema vorbereiten kann. Das Autorenteam ist sich aber bewusst, dass nicht alle Vokabeln sinnvoll eingedeutscht oder transponiert werden können. Begriffe wie »Open Access« oder »Hashtag« haben zwar bereits im fachlichen wie im alltäglichen Wortschatz Einzug gehalten, lassen sich aber weiterhin erläutern und verständlich erschließen. Zu diesem Zweck wurde ein umfangreiches Glossar der Fachbegriffe erarbeitet. Die entsprechenden Begriffe sind im Text mit einem $\rightarrow$ Pfeil gekennzeichnet.

Die Kapitel sind in sich geschlossen, also einzeln les- und verstehbar. Querverweise helfen dabei, vertiefende Informationen in benachbarten Kapiteln aufzufinden. Besonders gekennzeichnete Tipps im Text sollen Wichtiges pointiert herausheben. Zudem schließen die meisten Kapitel mit einem Katalog von Fragen und Anregungen, die zentrale Inhalte zusammenfassen oder für die eigene Anwendung in der Praxis inspirieren sollen. Hinweise auf weiterführende Literatur geben Ihnen die Möglichkeit, sich mit einzelnen Themen über diese Publikation hinaus vertiefend zu befassen.

Sollten Sie beim Lesen der Publikation beispielweise Best-Practice-Beispiele parallel online anschauen wollen, dann gelangen Sie im Buch über den zugeordneten QR-Code, den Sie nur mit einem mobilen Endgerät zu scannen brauchen, bzw. über die korrespondierenden Links im E-Book an die entsprechende Stelle im dynamischen Projekt-Portal.

\section{Inhaltlicher Aufbau}

Befasst man sich mit dem Museum im digitalen Zeitalter, gibt es viele Themen- und Aufgabenbereiche, die vor Veränderungen stehen. Nicht alle können in dieser Publikation erschöpfend dargestellt werden.

Kapitel 1 führt in den Wandel in der Kulturvermittlung durch Medieneinsatz in Ausstellungen sowie in die notwendige Erweiterung der Diskussion um Lerntheorien im Museum ein. Die Digitalisierung von Museumssammlungen und die strategische Entwicklung von Nutzungshorizonten für den internen und externen Gebrauch der Daten gehört zu den wichtigsten Aufgabenfeldern der Digitalisierung. Sie kann, aufgrund der Komplexität des Themas, in unserem MuseumsBaustein nur in Grundzügen dargelegt werden. Für dieses Kapitel konnten wir zwei Expertinnen gewinnen: Dr. Simone Mergen, Leiterin der Bildungsabteilung in der Stiftung Haus der Geschichte der Bundesrepublik Deutschland, die den Medieneinsatz aus der Sicht der Kulturvermittlung beleuchtet, und Dr. Antje Schmidt, Leiterin der digitalen Inventarisierung am Museum für Kunst und Gewerbe Hamburg, die sich mit den Potenzialen digitalisierter Museumssammlungen befasst.

Kapitel 2 thematisiert die gezielte Einbindung »neuer Medien« in die Kommunikation des Museums. Die Grundlage hierfür bietet die Entwicklung einer nachhaltigen und belastbaren digitalen Strategie, deren Konturen und Möglichkeiten aus- 
führlicher dargestellt werden. Im Fokus der Betrachtung stehen auch Aufgabenbereiche der Online-Kommunikation (Webseite, Social Media, E-Mail-Marketing oder Formen des digitalen Publizierens) sowie die Öffnung der Sammlung für eine Nachnutzung via Open Access. Das Digitale, insbesondere die sozialen Medien, verändert den Dialog mit dem Publikum. Wie dieser über Facebook, Twitter, Instagram und andere Kanäle gelingen kann, können Sie in Kapitel 3 nachlesen. Unsere Betrachtung fokussiert nicht nur die derzeit wesentlichen Kanäle oder schärft den Blick auf relevante Zielgruppen, sondern diskutiert auch Kommunikationsmodelle. Zudem beleuchten unsere Autoren Grundzüge einer Contentstrategie und die Potenziale neuer digitaler Kommunikations- und Eventformate.

In Kapitel 4 geht es um multimediale Elemente in der Vermittlung in Museen. Fest installierte Medienstationen und Audioguides sind in vielen Häusern seit geraumer Zeit selbstverständlicher Bestandteil des Angebots. Wenn wir aber heute über digitale Vermittlung reflektieren, kommen sehr schnell die Medienguides oder auch die sogenannten Apps auf den Tisch. Um hier mehr Licht in die Diskussion zu bringen, betrachten wir diese digitalen Vermittlungsinstrumente ausführlicher. Kapitel 5 befasst sich mit der Planung, dem Einbau und der Wartung der Medienanwendungen in Ausstellungen. Praxisorientierte Tipps sollen dazu beitragen, zentrale Fehler schon im Vorfeld zu vermeiden.

Wenn Museen ihren Bildungsauftrag ernst nehmen, müssen sie Informationen didaktisch schlüssig und in lebendiger Form vermitteln. Kapitel 6 beleuchtet daher das Themenspektrum von der Definition der Zielgruppen über Nutzerfreundlichkeit, Informationsdesign, Gestaltung bis hin zur Methode des Storytellings. Auch die dazu nötigen Hilfsmittel wie Drehbuch und Storyboard werden betrachtet. Kapitel 7 haben wir den »erweiterten Darstellungsformen « gewidmet, die mit den Möglichkeiten wie Virtual Reality, Augmented Reality oder 3D, aber auch der Künstlichen Intelligenz derzeit heftig in Bewegung sind. Da gerade in der Archäologie hier ambitionierte und hilfreiche Anwendungen entwickelt wurden, haben wir zwei Experten, Dott.ssa Francesca Morandini und Dr. Christof Flügel, um Fallstudien und eine Betrachtung über virtuelle Idealrekonstruktionen gebeten. Das Kapitel schließt auch den Bereich Gaming ein, der im Themenkomplex "Serious Games« zahlreiche Anwendungsbeispiele im Museumsbereich aufführt. 


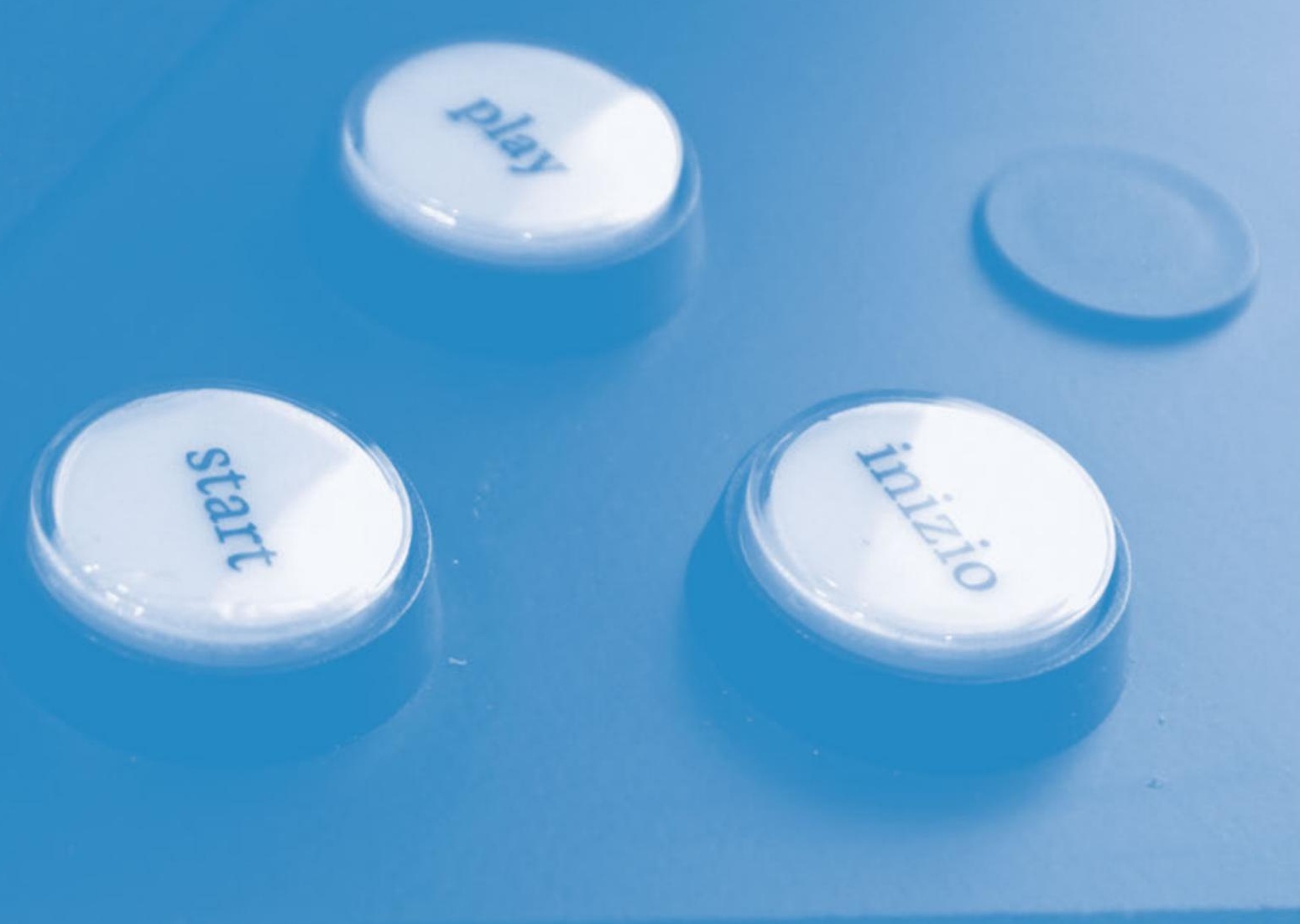

